\title{
An Emergency Dispatch Model Considering the Urgency of the Requirement for Reliefs in Different Disaster Areas
}

\author{
Liu Sheng ${ }^{1}$, Tan Wen-Jing ${ }^{1}$, Yu Guo-Dong ${ }^{1}$, Li Fei ${ }^{2}$ \\ ${ }^{1}$ State Key Laboratory of Mechanical Transmission, Chongqing University, Chongqing, 400030 \\ ${ }^{2}$ CISDI R and D Co., Ltd., Chongqing 401122 \\ lim@.cqu.edu.cn,tanwenjing1990@163.com,.vuguodong@rqu.edu.cn,feili@.cqu.edu.cn
}

Received: May 2015

Accepted: November 2015

\section{Abstract:}

Purpose: Frequent sudden-onset disasters which have threatened the survival of human and the development of society force the public to pay an increasing attention to emergency management. A challenging task in the process of emergency management is emergency dispatch of reliefs. An emergency dispatch model considering the urgency of the requirement for reliefs in different disaster areas is proposed in this paper to dispatch reliefs reasonably and reduce the effect of sudden-onset disasters.

Design/methodology/approach: Firstly, quantitative assessment on the urgency of the requirement for reliefs in different disaster areas is done by an evaluation method based on Fuzzy Comprehensive Evaluation and improved Evidence Reasoning which is proposed in this paper. And then based the quantitative results, an emergency dispatch model aiming to minimize the response time, the distribution cost and the unsatisfied rate of the requirement for reliefs is proposed, which reflects the requests of disaster areas under emergency, including the urgency of requirement, the economy of distribution and the equity of allocation. Finally, the Genetic Algorithm is improved based on the adaptive crossover and mutation probability function to solve the emergency dispatch model.

Findings: A case that the Y hydraulic power enterprise carries on emergency dispatch of reliefs under continuous sudden-onset heavy rain is given to illustrate the availability of the 
emergency dispatch model proposed in this paper. The results show that the emergency dispatch model meets the distribution priority requirement of disaster area with the higher urgency, so that reliefs are supplied more timely.

Research limitations/implications: The emergency dispatch model faced to large scale sudden-onset disasters is complex. The quantity of reliefs that disaster area requires and the running time of vehicles are viewed as available information, and the problem that how to obtain the information is not considered.

Practical implications: The emergency dispatch model considering the urgency of the requirement for reliefs in different disaster areas is applicable to a realistic emergency dispatch of reliefs under sudden-onset disasters and the research results is the foundation of further research on the problem of emergency management.

Originality/value: This paper proposes emergency dispatch model considering the urgency of the requirement for reliefs in different disaster areas which can meet the realistic requirement that the disaster area with the higher urgency has an priority to obtain the reliefs and make reliefs be distributed timely.

Keywords: sudden-onset disaster; urgency of the requirement for reliefs in different disaster areas; emergency dispatch; adaptive Genetic Algorithm

\section{Introduction}

The statistics reveal that 337 disasters occurred all over the world in 2013 , causing $\$ 135.4$ billion economic loss. The frequent natural disasters have threatened human survival and social development (International Red Cross, 2014). Emergency management under sudden-onset disasters has got increasing attention accordingly (Bozorgi-Amiri, Jabalameli \& Alinaghian, 2011). In the process of emergency management, the reasonable emergency dispatch scheme is critical to reduce the effect of sudden-onset disasters. It is not only the precondition to carry out emergency management effectively, but also the important guarantee to reduce casualties and property loss (Tian, Ma, Wang \& Wang, 2011). Therefore, it is that how to distribute reliefs timely, take advantage of the rescue value of reliefs and improve the efficiency of rescue becomes an important topic among domestic and foreign experts and scholars (Yuan \& Wang, 2009).

In 1984, Kemball-Cook and Stephenson (1984) proposed the concept of emergency dispatch of reliefs firstly, and analyzed the feature of relief dispatch under sudden-onset disasters. Afterwards, scholars at home and abroad did further research on emergency dispatch of reliefs 
and achieved a lot of significant results. Vitoriano, Ortuno and Tirado (2011) put forward the principles of emergency dispatch of reliefs, including timeliness, economy, fairness, priority, reliability, security, etc, and built a multi-objective programming model based on the principles. Combining the allocation of reliefs with the choice of vehicle routes, Chang, Wu and Lee (2014) built a model aiming to minimize the unsatisfied rate of requirement for reliefs, the time and the cost to distribute, and proposed a greedy-search-based multi-objective genetic algorithm to solve the model. Bozorgi-Amiri et al. (2011) studied the emergency dispatch of reliefs under stochastic environment by the method of robust optimization. A mixed-integer programming model was proposed to minimize the expected total cost, and an efficient solution approach based on particle swarm optimization was developed to solve the proposed mathematical model. Considering the evolution of the disasters, Li, Zhang and Zhu (2011) made a nonlinear programming model to minimize the total time of emergency dispatch of reliefs both in the process of primary disasters and secondary disasters, and designed a heuristic algorithm based on graph theory and linear programming. Wang, Ma and Ruan (2013) made an integer programming model with the goals to minimize the disaster loss and transportation cost in the consideration of the function relationship between disaster loss and disaster severity \& unsatisfied rate of requirement for reliefs, and improved genetic algorithm to solve the model. Barbarosoglu and Arda (2002) built a temporal and spatial network model which solved the problem of helicopter dispatch during the disaster rescue.

In summary, for the problem of emergency dispatch of reliefs, we can learn from the existing literatures that the research ideas that building multi-objective programming model and solving it with intelligent algorithm are tended to adopted. These literatures have an important instructive effect on the emergency dispatch decision. However, when built the emergency dispatch model, researchers often neglected the fact that there is difference of the requirement urgency for reliefs in different disaster areas. Although Vitoriano et al. (2011) and Pang, Liu and Wu (2012) took into account the difference when built the emergency dispatch model, but for the parameter of the requirement urgency for reliefs, they did not give the exact acquisition method. In fact, there is the conflict of relief requirement between different disaster areas under sudden-onset disaster. When the quantity of reliefs in the relief dispatching center can't meet the requirement of multiple disaster areas, if the urgency of the requirement for reliefs in different disaster areas is not considered or is not evaluated scientifically, it is difficult to satisfy the distribution priority requirement of disaster area with the higher urgency, and take advantage of the rescue value of reliefs accordingly.

The purpose of this study is to propose an emergency dispatch model considering the urgency of the requirement for reliefs in different disaster areas. For the urgency of the requirement for reliefs in different disaster areas, we propose an evaluation method based on Fuzzy Comprehensive Evaluation and improved Evidence Reasoning, which can help to solve the subjective, vague and uncertain information in the process of emergency dispatch. After proposing an emergency dispatch model, section 2 presents an improved Genetic Algorithm 
based on an adaptive crossover probability function and an adaptive mutation probability function to solve the model. In the section 3, the availability of the proposed emergency dispatch model is verified by a case. The final section concludes the paper.

\section{Modeling}

\subsection{Problem Description and Assumption}

This paper focuses on the problem of emergency dispatch which consists of relief dispatching center and multiple disaster areas. $S$ represents the relief dispatching center and $P=\left\{P_{1}, P_{2}, \ldots, P_{l}\right\}$ represents the set of disaster areas. When sudden-onset disasters occur, the quantity of reliefs that the disaster area $P_{j}$ requires is $M R_{j}$. At the same time, the total relief quantity of relief dispatching center $S$ is $M S$. The quantity of vehicles used to distribute reliefs is $v n$ and the number of the vehicle is $k(k=1,2, \ldots, v n)$. In addition, the running time of the vehicle through the path $\overrightarrow{r_{i j}}(i=0,1, \ldots, l ; j=1,2, \ldots, l)\left(i=0\right.$ represents relief dispatching center) is $T_{i j}(i=0,1, \ldots, l ; j=1,2, \ldots, l)$ and the running distance is $D_{i j}(i=0,1, \ldots, l ; j=1,2, \ldots, l)$. The transportation cost of per kilometer, the using cost of the vehicle and the load of the vehicle is $V C, F C$ and $L R$, respectively. Based on the description above, the key problem that this paper will solve is how to make the decision of relief allocation and vehicle route choice under sudden-onset disasters.

The emergency dispatch of reliefs faced to large scale sudden-onset disasters is complex. In order to study, some assumptions are set up as follow.

(1) The information of disaster areas such as the disaster grade, the number of victims, the quantity of reliefs that disaster area requires and so forth is timely available.

(2) The information concerning the running distance, the running time and the unit transportation cost from the relief dispatching center to every disaster area is available through GPS, previous data and experience.

(3) The load of each vehicle that is used to distribute reliefs in the relief dispatching center is the same.

(4) The reliefs that every disaster area requires should be distributed by only one vehicle. If the quantity of reliefs that disaster area requires overload the vehicle, the disaster area will be divided into two or plurality nodes.

(5) The distribution of disaster area with high urgency will be given priority.

(6) For the problem of emergency dispatch, the requirement of response time in the process of transporting reliefs from relief dispatching center to the disaster area is 
much higher than that in the return process. Meanwhile there is no problem of relief allocation in the return process either, so the return process is not considered in this paper.

\subsection{Evaluation on the Urgency of the Requirement for Reliefs in Different Disaster Areas Based on Fuzzy Comprehensive Evaluation and Improved Evidence Reasoning}

The urgency of the requirement for reliefs in different disaster areas is an important basis for the decision-making of emergency dispatch, and the accuracy of the evaluation result will directly affect the effective implementation of emergency dispatch scheme (Pang et al., 2012). Therefore, the evaluation method about the urgency of the requirement for reliefs in different disaster areas is studied before building the emergency dispatch model.

Evaluating on the urgency of the requirement for reliefs in different disaster areas is one of the important decisions in the process of emergency rescue. The method of multi-experts decision is generally adopted to ensure the objectivity and accuracy of the evaluation results. However, because of the emergency of rescue and the variability of environment, the information that decision makers obtain when sudden-onset disasters occur is fuzzy and uncertain, which leads to different even conflictive decisions from different experts. Thus, how to combine the decisions with conflicts is one of key questions in the process of evaluation. Thus, an evaluation method about the urgency of the requirement for reliefs in different disaster areas based on Fuzzy Comprehensive Evaluation and improved Evidence Reasoning is proposed. The evaluation process is shown in Figure 1. To begin with, based on the relevant research, an evaluation index system used to assess the urgency of the requirement for reliefs in different disaster areas is built. Next, the definition of evidence credibility and the calculation method of evidence conflict allocation weight are proposed. Based on this, the evidence combination rules are improved to combine the evaluation results from multi-experts and then the combination results become the basis of building fuzzy evaluation matrix. Thirdly, Analytic Hierarchy Process (AHP) is combined with Entropy Method to calculate the weight of every index. Finally, the evaluation result of every index and its weight are combined by the weighted composition operator to obtain the quantitative result of the urgency of the requirement for reliefs in different disaster areas. 


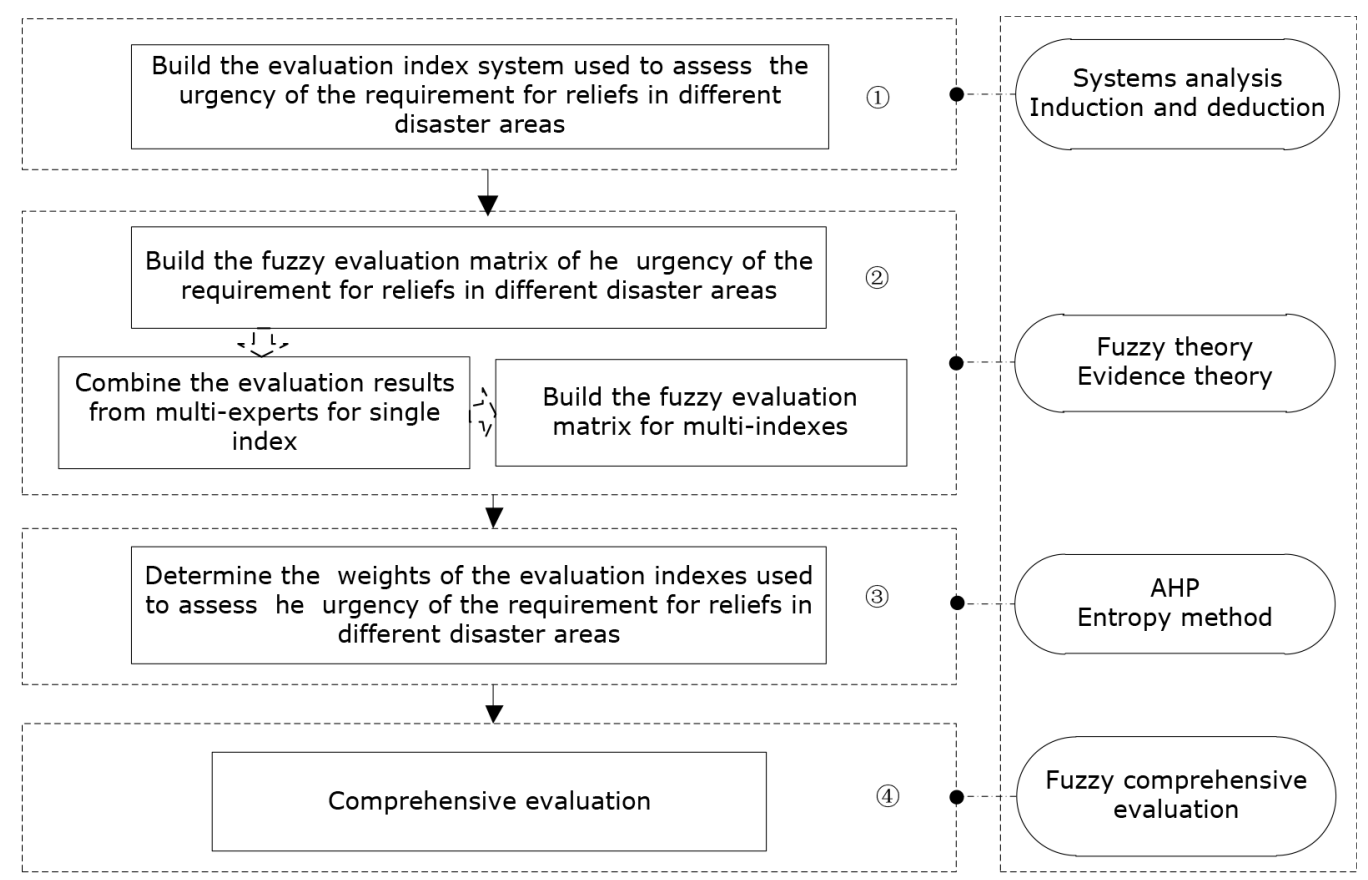

Figure 1. Evaluation process of the urgency of the requirement for reliefs in different disaster areas

\subsubsection{Evaluation Index System and Comment Set}

Through the systemic analysis on the characters of reliefs and the existing researches about the evaluation on the urgency of the requirement for reliefs in different disaster areas (Wang, Wang \& Ma, 2014; Guo \& Zhang, 2009; Shu,2012; Ge, Liu, Zhang \& Yu, 2010), we summarize the factors of the urgency of the requirement for reliefs in different disaster areas, and then build the evaluation index system. It is shown in Figure 2.

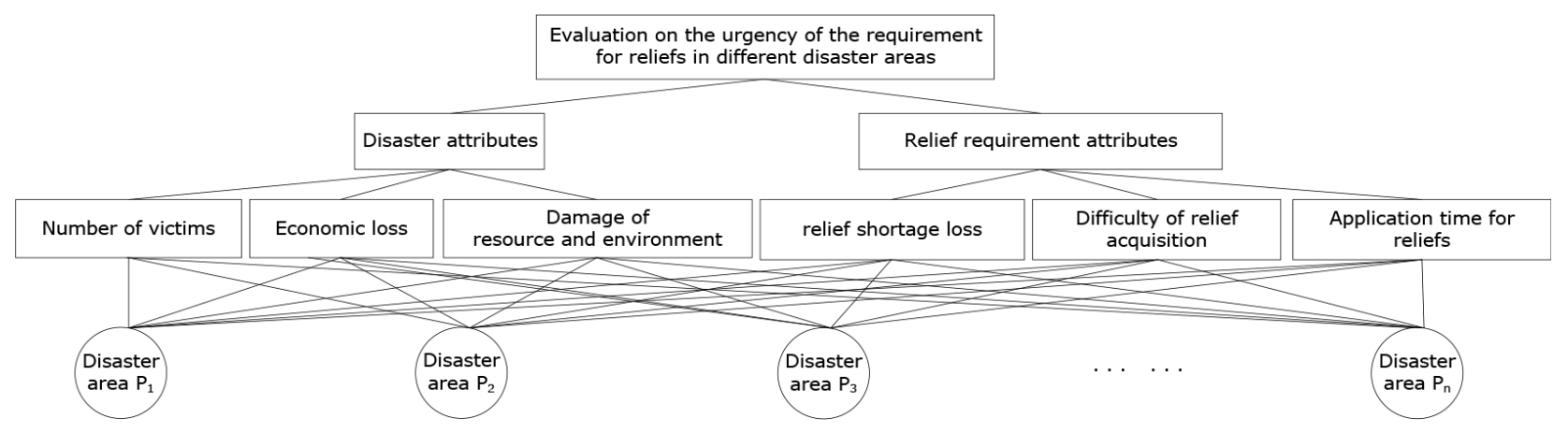

Figure 2. Evaluation index system used to assess the urgency of the requirement for reliefs in different disaster areas 
In order to make the evaluation results show difference and improve the efficiency of evaluation, for all the indexes used to assess the urgency of the requirement for reliefs in different disaster areas, 3 level evaluation scale is adopted, including high (0.9), medium (0.5), low $(0.1)$, which is presented by the comment set $C=\{0.9,0.5,0.1\}$.

\subsubsection{Fuzzy Evaluation Matrix Building Based on Improved Evidence Reasoning}

\subsubsection{Improvement of Evidence Combination Rules}

Evidence reasoning is an effective approach to combine uncertain information in the process of multi-expert decision-making. The D-S combination rule, also namely orthogonal sum, is the classical evidence combination rule (Jiang \& Yu, 2012). However, when D-S is used to combine the evidence with high conflicts, the combination results are against the facts (George \& Pal, 1996).

For the defect of D-S evidence combination rule, Lefevre, Colot and Vannoonrenberghe (2002) put forward the evidence combination model based on unified reliability function which is shown by Equation (1).

$$
m(A)=\sum_{\cap A_{f}=A} \prod_{1 \leq q \leq e n} m_{q}\left(A_{f}\right)+K \omega(A) \quad K=\sum_{\cap A_{f}=\varnothing} \prod_{1 \leq q \leq e n} m_{q}\left(A_{f}\right)
$$

Where Af $\in \Theta, K$ is the total conflict of evidence, $\omega(A)$ is the evidence conflict distribution coefficient and it meets equation $\sum_{A \in \Theta} \omega(A)=1$. The above model provides an effective idea to improve evidence combination rule and solve the problem of evidence combination with high conflicts. However, this model is applied to combine evidence under the assumption that all the evidence is equally important and the reliability of evidence is not in consideration, reducing the accuracy of the combination results to a certain extent. Besides, due to the lack of reasonable basis of evidence conflict distribution, there is no unified and certain method to determine the evidence conflict distribution coefficient $\omega(A)$ in exiting researches. Thus, based on the concepts and calculation methods of evidence distance, evidence similarity and the degree of support from other evidence that were proposed by Jousselme, Grenier and Bosse (2001), we raise the concept of evidence credibility. On the one hand, it corrects the original evidence and improves the reliability of the evidence. On the other hand, it can be the basis to determine the conflict evidence distribution coefficient and then provide a theoretical basis and solution to combine different ideas from multi-experts in the process of the evaluation on the urgency of the requirement for reliefs in different disaster areas. 
Definition 1. Let $m_{1}$ and $m_{2}$ be the two bodies of evidence in the same frame of discernment $\Theta,|\Theta|=N, 2^{\Theta}=\left\{A_{f} \mid f=1,2, \ldots, 2^{N}\right\}$. The distance between evidence $m_{1}$ and evidence $m_{2}$ is shown by Equation (2) (Jousselme et al., 2001).

$$
d\left(m_{1}, m_{2}\right)=\sqrt{\left(\overrightarrow{m_{1}}-\overrightarrow{m_{2}}\right)^{T} \underline{\underline{D}\left(\overrightarrow{m_{1}}-\overrightarrow{m_{2}}\right)}}
$$

Where $\overrightarrow{m_{q}}=\left[m_{q}\left(A_{1}\right), m_{q}\left(A_{2}\right), \ldots, m_{q}\left(A_{2^{N}}\right)\right](q=1,2)$. Let $\underline{\underline{D}}$ be the $2^{N} \times 2^{N}$ similarity metric matrix. The elements of the matrix are $D_{u v}=\left|A_{u} \cap A_{v}\right| /\left|A_{u} \cap A_{v}\right| A_{u}, A_{v} \in \Theta$.

Definition 2. Let $m_{1}, m_{2}, \ldots, m_{e n}$ be the en bodies of evidence in the same frame of discernment $\Theta$. For evidence $m_{q}(q=1,2, \ldots, e n)$, the degree of support from other evidence is shown by Equation (3) (Jousselme et al., 2001).

$$
\operatorname{Sup}\left(m_{q}\right)=\sum_{p=1, p \neq q}^{e n} \operatorname{Sim}\left(m_{p}, m_{q}\right)
$$

Where $\operatorname{Sim}\left(m_{p}, m_{q}\right)$ is evidence similarity between $m_{p}$ and $m_{q}$.

$$
\operatorname{Sim}\left(m_{p}, m_{q}\right)=1-d\left(m_{p}, m_{q}\right)
$$

Generally speaking, the more highly the evidence is supported by others, the more credible the evidence is. Otherwise the less credible the evidence is. Thus, the concept and calculation method of evidence credibility are put forward based on the degree of support from other evidence.

Definition 3. Let $m_{1}, m_{2}, \ldots, m_{e n}$ be the en bodies of evidence in the same frame of discernment $\Theta$, the credibility of evidence $m_{q}(q=1,2, \ldots$, en $)$ is shown by Equation (5).

$$
\operatorname{Crd}_{q}\left(m_{q}\right)=\frac{\operatorname{Sup}\left(m_{q}\right)}{\max _{1 \leq p \leq e n}\left[\operatorname{Sup}\left(m_{p}\right)\right]} \quad q=1,2, \cdots, \text { en }
$$

By normalizing, the relative credibility of evidence $m_{q}(q=1,2, \ldots, e n)$ is obtained which is shown by Equation (6).

$$
\operatorname{Crd}_{q}^{(r)}\left(m_{q}\right)=\frac{\operatorname{Crd}_{q}\left(m_{q}\right)}{\sum_{p=1}^{e n} \operatorname{Crd}_{p}\left(m_{p}\right)} \quad q=1,2, \cdots, e n
$$


Based on the credibility $\operatorname{Crd}_{q}\left(m_{q}\right)$ of evidence $m_{q}(q=1,2, \ldots, e n)$, the original evidence is corrected according to Equation (7).

$$
m_{q}^{\prime}(A)=\left\{\begin{array}{ll}
\operatorname{Crd}_{q}\left(m_{q}\right) \cdot m_{q}(A) & A \neq \Theta \\
1-\sum_{B \subset \Theta} \operatorname{Crd} d_{q}\left(m_{q}\right) \cdot m_{q}(B) & A=\Theta
\end{array} \quad q=1,2, \cdots,\right. \text { en }
$$

As Equation (7) shows, the correction of original evidence based on credibility reduces the influence of low credibility evidence on the combination result.

The evidence conflict distribution weight $\omega^{\prime}(A)$ is determined based on the relative credibility $\operatorname{Crd}_{q}^{(r)}\left(m_{q}\right)$ of evidence $m_{q}^{\prime}(q=1,2, \cdots, e n)$.

$$
\omega^{\prime}(A)=\sum_{q=1}^{e n} \operatorname{Cr} d_{q}^{(r)}\left(m_{q}\right) \cdot m_{q}^{\prime}(A)
$$

Based on the corrected evidence $m_{q}^{\prime}(q=1,2, \cdots, e n)$ and the evidence conflict distribution weight $\omega^{\prime}(A)$, the evidence combination model is improved by Equation (9).

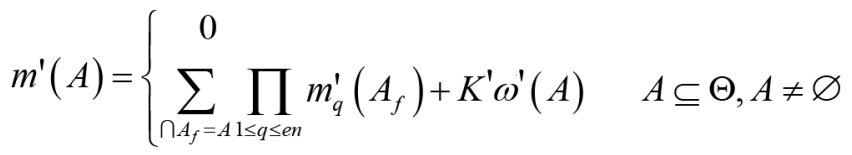

Where $K^{\prime}=\sum_{\cap A_{f}=\varnothing 1 \leq q \leq e n} \prod_{q}^{\prime}\left(A_{f}\right)$ refers to the total conflict of corrected evidence.

To verify the feasibility and effectiveness of the improved evidence combination method in this paper, the evidence combination method in this paper is compared with others that proposed by Yager (1987), Sun, Ye and Gu (2000) and Gao, Niu and Yang (2009).

For the economic loss that is an evaluation index, let the frame of discernment be $\Theta=\{$ high, medium, low $\}$, and the evidence from 4 experts be $m_{1}, m_{2}, m_{3}, m_{4}$ that are shown by Equation (10). Where the evidence $m_{2}$ is the disturbance evidence, while others support the element of high to a large extent. Combine the ideas from the 4 experts based on the evidence combination methods proposed by Yager (1987), Sun et al. (2000) and Gao et al. (2009) and this paper respectively. The combination results are shown in Table 1.

$$
\begin{array}{ll}
m_{1}(\text { high })=0.5, m_{1}(\text { medium })=0.2, m_{1}(\text { low })=0.3 & m_{2}(\text { high })=0.0, m_{2}(\text { medium })=0.9, m_{2}(\text { low })=0.1 \\
m_{3}(\text { high })=0.6, m_{3}(\text { medium })=0.1, m_{3}(\text { low })=0.3 & m_{4}(\text { high })=0.9, m_{4}(\text { medium })=0.1, m_{4}(\text { low })=0.1
\end{array}
$$

As shown in Table 1, the results obtained by the methods which are proposed by Yager (1987) and Sun et al. (2000) respectively are against the visual analysis results. While the results obtained by the methods proposed by Gao et al. (2009) and this paper respectively are reasonable. In other words, the methods which are put forward by Gao et al. (2009) and this paper are robust. 


\begin{tabular}{|l|r|r|r|r|}
\hline \multirow{2}{*}{\multicolumn{1}{c|}{ Method }} & \multicolumn{4}{|c|}{ Evidence } \\
\cline { 2 - 5 } & \multicolumn{1}{c|}{$\boldsymbol{m}$ (high) } & \multicolumn{1}{c|}{ m(medium $)$} & \multicolumn{1}{c|}{$\boldsymbol{m}($ low $)$} & \multicolumn{1}{c|}{$\boldsymbol{m ( \Theta )}$} \\
\hline Yager (1987) & 0.3341 & 0.2304 & 0.1416 & 0.2939 \\
\hline Sun et al. (2000) & 0 & 0.0018 & 0.0009 & 0.9973 \\
\hline Gao et al. (2009) & 0.6190 & 0.2450 & 0.1360 & 0 \\
\hline This paper & 0.5670 & 0.2306 & 0.2024 & 0
\end{tabular}

Table 1. Comparative analysis on the evidence combination results

In order to analyze the convergence of the method in this paper, we calculate and get the results which are shown in Table 2.

\begin{tabular}{|l|c|c|c|c|}
\hline \multirow{2}{*}{ Method } & \multicolumn{4}{|c|}{ Evidence } \\
\cline { 2 - 5 } & & $\boldsymbol{m}_{\mathbf{1}}, \boldsymbol{m}_{\mathbf{2}}$ & $\boldsymbol{m}_{\mathbf{1}} \sim \boldsymbol{m}_{3}$ & $\boldsymbol{m}_{\mathbf{1}} \sim \boldsymbol{m}_{4}$ \\
\hline \multirow{3}{*}{ GAO She-sheng et al.(2009) } & $m$ (high) & 0.0860 & 0.3070 & 0.6190 \\
\cline { 2 - 5 } & $m$ (medium) & 0.8040 & 0.5570 & 0.2450 \\
\cline { 2 - 5 } & $m($ low $)$ & 0.1100 & 0.1360 & 0.1360 \\
\hline \multirow{3}{*}{ This paper } & $m($ high $)$ & 0.1975 & 0.4102 & 0.5670 \\
\cline { 2 - 5 } & $m$ (medium) & 0.6145 & 0.2201 & 0.2306 \\
\cline { 2 - 5 } & $m$ (low) & 0.1880 & 0.2402 & 0.2024 \\
\hline
\end{tabular}

Table 2. Comparative analysis on the convergence of evidence combination methods

As shown in Table 2, the results obtained by the method which is proposed by Gao et al. (2009) are inconsistent with the visual analysis results until the 4 bodies of evidence are all combined. While the results obtained by the method in this paper are consistent with the visual analysis results when the first 3 bodies of evidence are combined. Thus, the method in this paper has better astringency.

\subsubsection{Fuzzy Evaluation Matrix Building}

Based on the above evidence combination method, the ideas from multi-experts in the process of the evaluation on the urgency of the requirement for reliefs in different disaster areas are combined effectively, and the fuzzy evaluation matrix is built on the basis of the above combination results to improve the reliability of evaluation results.

Assume that there are $e n(e n \geq 2)$ experts who evaluate on $I_{s}(s=1,2, \ldots, 6)$ that are the evaluation indexes of the urgency of the requirement for reliefs in different disaster areas 
and the comment set is $\left\{C_{f}(f=1,2,3)\right\}$. Let the evaluation results from expert $E_{q}(q=1,2, \ldots$, en $)$ be $p_{E_{q}}^{I_{s}}=\left\{C_{1}: p_{E_{q}}^{I_{s}}\left(C_{1}\right) ; C_{2}: p_{E_{q}}^{I_{s}}\left(C_{2}\right) ; C_{3}: p_{E_{q}}^{I_{s}}\left(C_{3}\right)\right\}\left(\sum_{f=1}^{c n} p_{E_{q}}^{I_{s}}\left(C_{f}\right)=1\right)$

Where $p_{E_{q}}^{I_{s}}\left(C_{f}\right)$ refers to the probability that the comment of the index $I_{s}$ made by expert $E_{q}$ is $C_{f}$. Let $m_{E_{q}}^{I_{s}}\left(C_{f}\right)=p_{E_{q}}^{I_{s}}\left(C_{f}\right)$. In other words, the evaluation results from en experts are expressed as the en bodies of evidence in the same frame of discernment $\Theta$, where $2^{\Theta}=\left\{C_{f} \mid f=1,2,3\right\}$. Based on the above evidence combination method, the combination results $m^{\iota_{s}}\left(C_{f}\right)(s=1,2, \ldots, 6 ; f$ $=1,2,3)$ of $e n$ bodies of evidence are got. $m^{I_{s}}\left(C_{f}\right)(s=1,2, \ldots, 6 ; f=1,2,3)$ is defined as Membership Degree Vector, where the index $I_{s}$ belongs to the comment set $\left\{C_{f}(f=1,2,3)\right\}$. By evaluating all the indexes in turn, the fussy evaluation matrix $R=\left[m^{I_{s}}\left(C_{f}\right)\right]_{6 \times 3}(s=1,2, \ldots, 6 ; f=1,2,3)$ is built.

\subsubsection{Index Weight Calculation Based on AHP and Entropy Method}

To ensure the accuracy of the evaluation results, the above-mentioned indexes should be weighted according to their relative importance for the urgency of the requirement for reliefs in different disaster areas. We make full use of the advantages of AHP and Entropy Method and adopt the way which combinations subjective method with objective method to calculate the weight of every index.

Firstly, the subjective weight $\alpha_{s}(s=1,2, \ldots, 6)$ of index $I_{s}(s=1,2, \ldots, 6)$ is got through the method of AHP described by Guo, Tong, Shao, Wang and Zheng. (2013). Then, the objective weight $\beta_{s}(s=1,2, \ldots, 6)$ of index $I_{s}(s=1,2, \ldots, 6)$ is got through Entropy Method described by Guo, Pu, Gao and Zhang (2014). Lastly, the subjective weight is combined with the objective weight through the geometric mean method which is shown by Equation (11).

$$
u_{s}=\sqrt{\alpha_{s} \cdot \beta_{s}}
$$

Normalize $u_{s}$ by Equation (12) to get the comprehensive weight set $U^{\prime}=\left(u_{1}^{\prime}, u_{2}^{\prime}, \cdots, u_{6}^{\prime}\right)$.

$$
u_{s}^{\prime}=\frac{u_{s}}{\sum_{s=1}^{i n} u_{s}}
$$

\subsubsection{Comprehensive Evaluation}

We combine the identified weights of all the indexes $U=\left(u_{1}^{\prime}, u_{2}^{\prime}, \cdots, u_{6}^{\prime}\right)$ with the fuzzy evaluation matrix $R=\left[m^{I_{s}}\left(C_{f}\right)\right]_{6 \times 3}(s=1,2, \ldots, 6 ; f=1,2,3)$ by the weighted composition operator to obtain 
the fuzzy evaluation vector $X_{j}$ of the urgency of the requirement for reliefs in the disaster area $P_{j}(j=1,2, \ldots, l)$.

$$
X_{j}=U^{T} R
$$

In order to make the evaluation results quantitative, we calculate the value $\chi_{j}^{\prime}$ of the urgency of the requirement for reliefs in the disaster area $P_{j}(j=1,2, \ldots, l)$ by Equation (14).

$$
\chi_{j}^{\prime}=C^{T} X_{j}
$$

Where $C=\{0.9,0.5,0.1\}$ presents the comment set.

\subsection{Emergency Dispatch Model Formulation Based on the Urgency of the Require- ment for Reliefs in Different Disaster Area}

In the consideration of the requirements of promptness, economy and fairness of emergency dispatch, a multi-objective integer programming model is built as follows.

Objective functions: $\min =\left\{f_{1}, f_{2}, f_{3}\right\}$

$$
\begin{gathered}
f_{1}=\sum_{k=1}^{v n} \sum_{j=1}^{l} \chi_{j}^{\prime} \cdot A T_{j}^{k} \\
f_{2}=\sum_{k=1}^{v n} \sum_{i=0}^{l} \sum_{j=1}^{l} D_{i j} \cdot V C \cdot x_{i j k}+\sum_{k=1}^{v n} F C \cdot V_{k} \\
f_{3}=\sum_{k=1}^{v n} \sum_{j=1}^{l} \chi_{j}^{\prime}\left(1-\frac{M_{j}}{y_{j k}}\right)
\end{gathered}
$$

Constraints:

$$
\begin{gathered}
V_{k}=\left\{\begin{array}{cc}
1 & \sum_{i=0}^{l} \sum_{j=1}^{l} x_{i j k} \geq 1 \quad k=1,2, \cdots, v n \\
0 & \text { others }
\end{array}\right. \\
x_{i j k}=\left\{\begin{array}{rr}
\sum_{k=1}^{v n} V_{k} \leq v n \\
\text { the vehicle } k \text { goes through the route } \overrightarrow{r_{i j}} \\
0 \quad \begin{array}{r}
\text { and distributes the reliefs to the disaster area } P_{j} \\
\text { others }
\end{array} \\
A T_{j}^{k} \geq A T_{i}^{k}+T_{i j}-\left(1-x_{i j k}\right) M \quad i=0,1, \cdots, l ; j=1,2, \cdots, l ; k=1,2, \cdots, v n
\end{array}\right.
\end{gathered}
$$




$$
\begin{gathered}
\sum_{j=1}^{l} x_{i j k} \leq 1 \quad i=0,1, \cdots, l ; k=1,2, \cdots, v n \\
\sum_{i=0}^{l} \sum_{k=1}^{v n} x_{i j k}=1 \quad j=1,2, \cdots, l \\
\sum_{j=1}^{l} x_{0 j k}=1 \quad V_{k}=1 ; k=1,2, \cdots, v n \\
x_{h 0 k}=1 \quad V_{k}=1 ; h=1,2, \cdots, l ; k=1,2, \cdots, v n \\
\sum_{i=0}^{l} x_{i h k}-\sum_{j=1}^{l} x_{h j k}=0 \quad \sum_{i=0}^{l} x_{i h k}-x_{h 0 k}=0 \\
y_{j k} \geq 0 \text { and } y_{j k} \text { is integer } \\
\sum_{k=1}^{v n} \sum_{j=1}^{l} y_{j k} \leq M S \\
\sum_{j=1}^{l} y_{j k} \leq L R \quad k=1,2, \cdots, v n ; h=1,2, \cdots, l \\
k n=1,2, \cdots, v n \\
i \neq j
\end{gathered}
$$

The objective function $f_{1}$ represents the minimization of the emergency response time. Where, parameter $A T_{j}^{k}$ represents the taken time that the rescue vehicle $k$ arrives to the disaster area $P_{j}$. Considering there is difference of relief requirement urgency between different disaster areas, we multiply $\chi_{j}^{\prime}$ by $A T_{j}$ to obey the dispatching rule that the disaster area with highest urgency should be distributed first. The objective function $f_{2}$ represents the minimization of distribution cost which involves the transportation cost and the vehicle leasing cost. The objective function $f_{3}$ represents the minimization of the unsatisfied rate of reliefs which the disaster area requires. Due to the difference of relief requirement urgency between different disaster areas, the damages caused by a lack of reliefs are different. Thus, we multiply $\chi_{j}^{\prime}$ by $\left(1-M / y_{j k}\right)$ to show the fairness of relief distribution. Next, we analyze the constraints. In Equation (18), $V_{k}$ is the decision variable that represents whether the vehicle $k$ takes part in the rescue operation; Equation (19) represents that the quantity of the vehicles used does not outnumber the quantity of the vehicles in the relief dispatching center; In Equation (20), $x_{i j k}$ is another decision variable that represents whether the vehicle $k$ distributes the reliefs to the disaster area $P_{j}$ through the route $\overrightarrow{r_{i j}}$; Equation (21) represents the calculation method of the taken time that rescue vehicle $k$ arrives to the disaster area $P_{j}$; Equation (22) represents that 
the vehicle $k$ can go out from only one node (the relief dispatching center or the disaster area) one time; Equation (23) represents that there's one and only one vehicle to distribute the reliefs for the disaster area $P_{j}$; Equation (24) represents that the vehicle taking part in the emergency dispatch must set out from the relief dispatching center; Equation (25) and Equation (26) represent the continuity of the vehicle running routes. Because the return processes of vehicles are not taken into consideration in this paper, Equation (26) represents the virtual routes of vehicles to meet the continuity of routes; In Equation (27), $y_{j k}$ is the third decision variable that represents the quantity of reliefs distributed by vehicle $k$ for the disaster area $P_{j}$; Equation (28) represents that the quantity of reliefs exported from the relief dispatching center does not outnumber the total relief supply; Equation (29) represents that the actual load of the vehicle $k$ does not outnumber the maximum load limit; Equation (30) represents that the quantity of reliefs distributed in the disaster area $P_{j}$ does not outnumber the requirement quantity; Equation (31) represents that the running route of vehicle $k$ does not repeat in a certain node (the relief dispatching center or a disaster area).

\section{Algorithm}

The emergency dispatch of reliefs is a combinational optimization problem of multi-disaster areas and multi-vehicles. Genetic Algorithm which simulates the biology evolution process has been proved to be a high efficient method to search optimization solution. It has strong global searching ability and is effective to solve the problems of combinational optimization (Bao, Yang, Li, Liu \& Liu, 2014). However, the classical genetic algorithm is apt to fall into the locally optimal solution, leading to the phenomenon of prematurity (Liu \& Zhou, 2009). For the combinational optimization features of the emergency dispatch model, we put forward a coding method based on the disaster areas and improve the genetic algorithm based on the self-adaption crossover probability functions and self-adaption mutation probability functions to solve the model. The flowchart in Figure 3 shows the steps of the improved algorithm. 


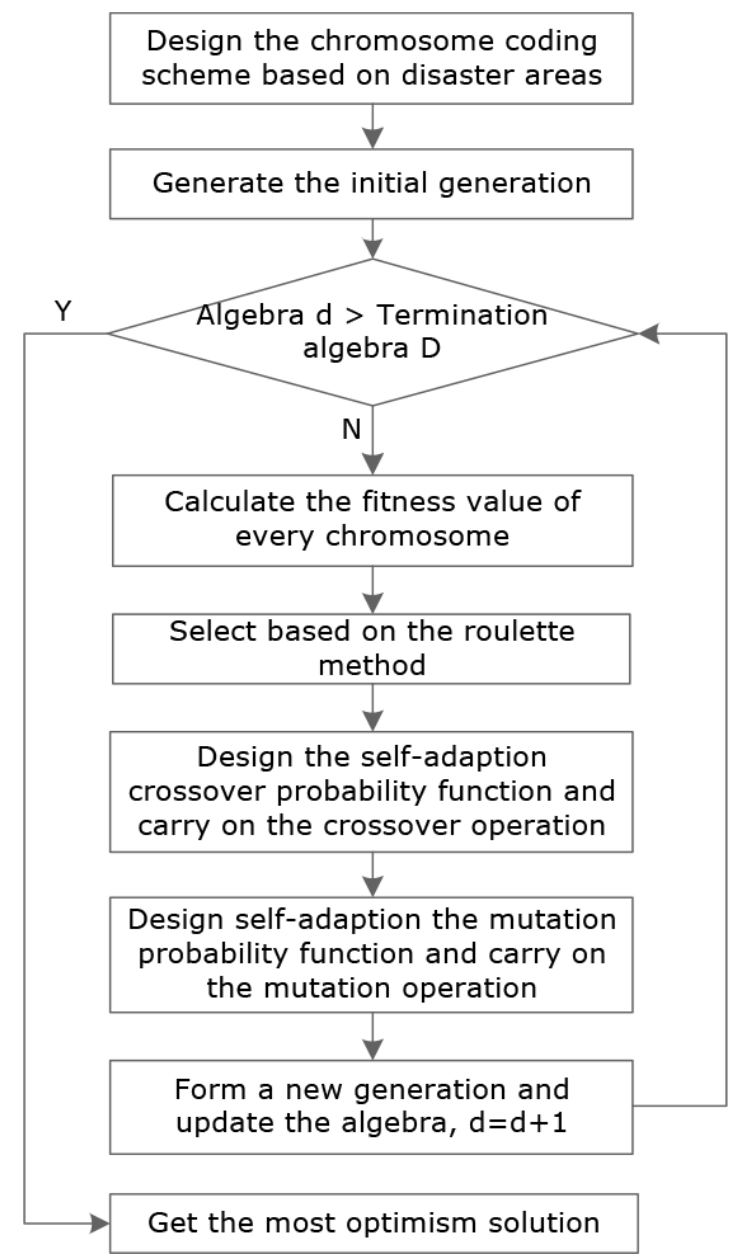

Figure 3. Process of the self-adaption Genetic Algorithm

Step 1. Chromosome coding. Considering the features of the problem studied in this paper and the existing coding schemes for the relief dispatch model (Wang, Yang \& Xu, 2009), we propose a 3-layers coding strategy that contains the vehicle number, the vehicle routing and the quantity of reliefs. The scheme is as follow: the chromosome is denoted by the gene sequence $\left(G_{1}, G_{2}, \ldots, G_{j} ; j=1,2, \ldots, l\right)$. Each gene $G_{j}$ represents a disaster area $P_{j} ; G_{j}$ is composed of three layers and each layer is coded by the float-point encoding method. Where the first layer represents the number of the rescue vehicle $k$; the second layer represents the starting point of the running route $\overrightarrow{r_{i j}}$ of the vehicle $k$; the third layer represents the quantity of reliefs that the vehicles $k$ distributes to the disaster area $P_{j}$.

Figure 4 represents an available chromosome coding scheme. This scheme shows that the running routes of the vehicle 1 is $0 \rightarrow 1 \rightarrow 4$, and the quantity of reliefs distributed by vehicle 1 for the disaster area $P_{1}$ and $P_{4}$ respectively is 3 and 1 . In a similar way, we get the running routes of other vehicles and the distribution quantity of reliefs for other disaster areas. 


\begin{tabular}{|c|c|c|c|c|c|c|c|c|c|}
\hline Disaster points & 1 & 2 & 3 & 4 & 5 & 6 & 7 & 8 & 9 \\
\hline Numbers of vehicles & 1 & 2 & 3 & 1 & 4 & 5 & 6 & 4 & 6 \\
\hline $\begin{array}{l}\text { Starting points of } \\
\text { routes }\end{array}$ & 0 & 0 & 0 & 1 & 8 & 0 & 0 & 0 & 7 \\
\hline $\begin{array}{l}\text { Quantity of material } \\
\text { distributed }\end{array}$ & 3 & 5 & 4 & 1 & 2 & 4 & 2 & 2 & 1 \\
\hline
\end{tabular}

Figure 4. An available chromosome coding scheme

Step 2. The generation of the initial population. The size of the population popsize is determined according to the number of the disaster areas. The generation of chromosomes in the initial population is divided into three phases. Firstly, the first layer codes are generated randomly. Secondly, if the first layer codes show that one vehicle distributes reliefs to multiple disaster areas, the order of distribution will be generated randomly under the constraint conditions (18) (26). Then the second layer codes are generated. Finally, the quantity of reliefs distributed to the disaster areas are generated randomly under the constraint conditions (27) (31). Then the third codes are generated.

Step 3. Fitness functions design. The emergency dispatch model proposed in this paper is a multi-objective programming model. The usual solving methods for it are the methods of Pareto and Weight Coefficient. We can get a group of non-dominated solution set through the method of Pareto, but if we want to get the optimal solution, we still make another decision. While we can converge to a global optimal solution through the method of Weight Coefficient, and get the global optimal solution without another decision. Therefore, the method of weight coefficient is adopted to solve the emergency dispatch model in this paper.

Firstly, the value of every objective function is dealt with the Dimensionless Method according to the method that is proposed by Wang et al. (2014), and the transformation formula is

$$
\Phi_{c}(\psi)=\frac{f_{c}\left(\psi_{c}^{\max }\right)-f_{c}(\psi)}{f_{c}\left(\psi_{c}^{\max }\right)-f_{c}\left(\psi_{c}^{\min }\right)} \quad c=1,2,3
$$

In Equation (32), $\psi_{c}^{\max }$ and $\psi_{c}^{\min }$ are the solutions when the objective function $c$ gets the maximum value and minimum value respectively. Then, the optimum weights of the objective functions $\lambda_{i}(i=1,2,3)$ are obtained according to the method that is proposed by Zhang, Zhang, Luo and Xie (2014) and the above multi-objective problem is transformed into the following single objective problem by the method of linear weighting.

$$
\begin{aligned}
& \min F=\min \left(\lambda_{1} \Phi_{1}+\lambda_{2} \Phi_{2}+\lambda_{3} \Phi_{3}\right) \\
& \text { s.t. } \quad E q .(4)-(13)
\end{aligned}
$$

The fitness function is designed as follows. 


$$
\text { Fit }=\frac{1}{F}
$$

Step 4. Selecting operation. The selecting operation for the initial population is carried on based on the roulette method. On the assumption that the scale of the initial population is popsize and the fitness value of the chromosome $b$ is Fit ${ }_{b}$, the probability of the chromosome selected is

$$
\lambda_{b}=F_{b} / \sum_{b=1}^{\text {popsize }} \text { Fit }_{b}
$$

Step 5. Self-adaption crossover operation. The strategy of two-point crossover is adopted to increase the diversity of populations and avoid the prematurity and stagnating of the algorithm in this paper. In order to increase the efficiency of the crossover operation, only the first layer codes of the chromosome are carried on the crossover operation, the second layer codes and the third layer codes are generated by the same method as step 2 . The crossed chromosome must meet all the constraints. On the assumption that the quantity of the reliefs in $M S$ (the relief dispatching center) is 25 , and the $L R$ (the load of vehicle) is 5 . The diagram of the twopoint crossover operation is shown in Figure 5.

Besides, in order to increase the search ability of the crossover operator, self-adaption crossover probability function Pcb is proposed in this paper to guide the crossover operation. The basic idea of the self-adaption crossover is that the different chromosomes are given the different crossover probability. To protect the individual with high fitness value, it should be given the lower crossover probability, while the crossover probability of the individual with low fitness value should be added. It can avoid the damage of the excellent chromosomes and guarantee the diversity of the population by the method of self-adaption crossover (Liu \&

\begin{tabular}{|c|c|c|c|c|c|c|c|c|c|}
\hline Disaster areas & 1 & 2 & 3 & 4 & 5 & 6 & 7 & 8 & 9 \\
\hline Numbers of vehicles & 1 & 2 & 3 & 1 & 4 & 5 & 4 & 6 & 6 \\
\hline $\begin{array}{l}\text { Starting points of } \\
\text { routes }\end{array}$ & 0 & 0 & 0 & 1 & 0 & 0 & 5 & 0 & 8 \\
\hline $\begin{array}{l}\text { Quantity of reliefs } \\
\text { distributed }\end{array}$ & 3 & 4 & 4 & 2 & 3 & 5 & 1 & 2 & 1 \\
\hline Disaster areas & 1 & 2 & 3 & 4 & 5 & 6 & 7 & 8 & 9 \\
\hline Numbers of vehicles & 1 & 2 & 3 & 1 & 4 & 5 & 6 & 4 & 6 \\
\hline $\begin{array}{l}\text { Starting points of } \\
\text { routes }\end{array}$ & 0 & 0 & 0 & 1 & 8 & 0 & 0 & 0 & 7 \\
\hline $\begin{array}{l}\text { Quantity of reliefs } \\
\text { distributed }\end{array}$ & 3 & 5 & 4 & 1 & 2 & 4 & 2 & 2 & 1 \\
\hline
\end{tabular}
Zhou, 2009). The value of the crossover probability Pcb is shown in Equation (36).

\begin{tabular}{|c|c|c|c|c|c|c|c|c|c|}
\hline Disaster areas & 1 & 2 & 3 & 4 & 5 & 6 & 7 & 8 & 9 \\
\hline Numbers of vehicles & 1 & 2 & 3 & 1 & 4 & 5 & 6 & 6 & 6 \\
\hline $\begin{array}{l}\text { Starting points of } \\
\text { routes }\end{array}$ & 0 & 0 & 0 & 1 & 0 & 0 & 8 & 0 & 7 \\
\hline $\begin{array}{l}\text { Quantity of reliefs } \\
\text { distributed }\end{array}$ & 3 & 4 & 4 & 2 & 3 & 5 & 1 & 2 & 1 \\
\hline Disaster areas & 1 & 2 & 3 & 4 & 5 & 6 & 7 & 8 & 9 \\
\hline Numbers of vehicles & 1 & 2 & 3 & 1 & 4 & 5 & 4 & 4 & 6 \\
\hline $\begin{array}{l}\text { Starting points of } \\
\text { routes }\end{array}$ & 0 & 0 & 0 & 1 & 0 & 0 & 5 & 7 & 0 \\
\hline $\begin{array}{l}\text { Quantity of reliefs } \\
\text { distributed }\end{array}$ & 3 & 5 & 4 & 1 & 2 & 4 & 1 & 2 & 3 \\
\hline
\end{tabular}

Figure 5. Diagram of the two-point crossover operation 


$$
P_{c b}= \begin{cases}P_{c \text { min }}-\frac{F i t_{\max }-F i t_{b}}{F i t_{\max }-F i t_{\min }}\left(P_{c \max }-P_{c \min }\right) & F i t_{b}>\overline{F i t} \\ P_{c \text { min }}+\frac{F i t_{\max }-F i t_{b}}{F i t_{\max }-F i t_{\text {min }}}\left(P_{c \max }-P_{c \min }\right) & F i t_{b} \leq \overline{F i t}\end{cases}
$$

Where $F i t_{\max }, F i t_{\min }$ and $\overline{\text { Fit }}$ are the maximum fitness value, the minimum fitness value and the average fitness value respectively; Fit $_{b}$ is the fitness value of each crossover individual; $P_{c \max }$ and $P_{c \min }$ are the maximum crossover probability and the minimum crossover probability respectively that have been set.

Step 6. Self-adaption mutation operation. The strategy of random exchange mutation is adopted in this paper. Two genes of the chromosome are chose randomly and only the first layer codes of them are exchanged. The second layer codes and the third layer codes are generated by the same method as step 2 . The mutated chromosome must meet all the constraint conditions. On the assumption that the quantity of the reliefs in $M S$ (the relief dispatching center) is 25 , and the $L R$ (the load of vehicle) is 5 . The diagram of the random exchange mutation operation is shown in Figure 6.

\begin{tabular}{|c|c|c|c|c|c|c|c|c|c|}
\hline Disaster areas & 1 & 2 & 3 & 4 & 5 & 6 & 7 & 8 & 9 \\
\hline Numbers of vehicles & 1 & 2 & 3 & 1 & 4 & 5 & 6 & 4 & 6 \\
\hline $\begin{array}{l}\text { Starting points of } \\
\text { routes }\end{array}$ & 0 & 0 & 0 & 1 & 8 & 0 & 0 & 0 & 7 \\
\hline $\begin{array}{l}\text { Quantity of reliefs } \\
\text { distributed }\end{array}$ & 3 & 5 & 4 & 1 & 2 & 4 & 2 & 2 & 1 \\
\hline
\end{tabular}

\begin{tabular}{|c|c|c|c|c|c|c|c|c|c|}
\hline Disaster areas & 1 & 2 & 3 & 4 & 5 & 6 & 7 & 8 & 9 \\
\hline Numbers of vehicles & 1 & 2 & 31 & 6 & 4 & 5 & 1 & 4 & 6 \\
\hline $\begin{array}{l}\text { Starting points of } \\
\text { routes }\end{array}$ & 0 & 0 & 0 & 9 & 8 & 0 & 1 & 0 & 0 \\
\hline $\begin{array}{l}\text { Quantity of reliefs } \\
\text { distributed }\end{array}$ & 3 & 5 & 4 & 1 & 2 & 4 & 2 & 2 & 1 \\
\hline
\end{tabular}

Figure 6. Diagram of the random exchange mutation operation

Similarly, the value of the mutation probability $P_{m b}$ is as follows.

$$
P_{m b}= \begin{cases}P_{m \text { max }}-\frac{F i t_{\text {max }}-F i t_{b}^{\prime}}{F i t_{\text {max }}-F i t_{\text {min }}}\left(P_{m \text { max }}-P_{m \text { min }}\right) & F i t_{b}^{\prime}>\overline{F i t} \\ P_{m \text { max }}+\frac{F i t_{\text {max }}-F i t_{b}^{\prime}}{F i t_{\text {max }}-F i t_{\text {min }}}\left(P_{m \text { max }}-P_{m \text { min }}\right) & F i t_{b}^{\prime} \leq \overline{F i t}\end{cases}
$$

Where $F i t_{\max }, F i t_{\min }$ and $\overline{F i t}$ are the maximum fitness value, the minimum fitness value and the average fitness value respectively; $F i t_{t}^{\prime}$ is the fitness value of each mutation individual; $P_{m \max }$ and $P_{m \min }$ are the maximum mutation probability and the minimum mutation probability respectively that have been set.

Till now, the first step of iterative process of self-adaption genetic algorithm is finished, forming a new generation. Then, we update the algebra, $d=d+1$, and loop iteration until $d$ becomes greater than the termination algebra $D$ defined beforehand. At this time, the 
emergency dispatch scheme considering the urgency of the requirement for reliefs in different disaster areas is got.

\section{Case}

In the summer of 2013, the central and western regions of Sichuan Province were hit by the continuous heavy rain, which caused huge damages in many hydroelectric power stations and had a serious impact on the local daily electricity supply. Thus, the $\mathrm{Y}$ hydraulic power enterprise carried on the emergency dispatch of reliefs timely and reasonably to accelerate post-disasters recovery and reconstruction. Based on the background of the above event, we design a simulation example to verify the feasibility and validity of the emergency dispatch model in this paper.

Cables are regarded as the distributed reliefs. The storehouse $B$ is chosen as the relief dispatching center and its number is 0 . Ten hydroelectric power stations $Y X, W L, W C, W J, P J$, $\mathrm{CD}, \mathrm{QB}, \mathrm{JT}, \mathrm{DY}, \mathrm{MY}$ are chosen as the disaster areas $P_{1}, P_{2}, \ldots, P_{10}$ and their numbers are $1,2, \ldots$, 10 respectively. The transportation network topology of the relief dispatching center and all the disaster areas is shown in Figure 7. The quantity of the vehicles used to distribute reliefs in the relief dispatching center is $6(v n=6)$; the load of the vehicle is $25(L R=25)$; the using cost of the vehicle is $1000(F C=1000)$; the transportation cost of per kilometer is $20(V C=20)$; the running distance $D_{i j}$ and the running time $T_{i j}$ of the vehicle through the path $\overrightarrow{r_{i j}}(i=0,1, \ldots, l ; j=$ $1,2, \ldots, l)$ are shown in Table 3 , in which the units of time and distance are $\mathrm{h}$ and $\mathrm{km}$ respectively. The quantity of cables that the relief dispatching center can supply is 80 ( $M S=$ $80)$ and the quantity of cables that disaster areas require $M R_{j}(j=1,2, \ldots, 10)$ is shown in Table 4, in which the unit is $t$.

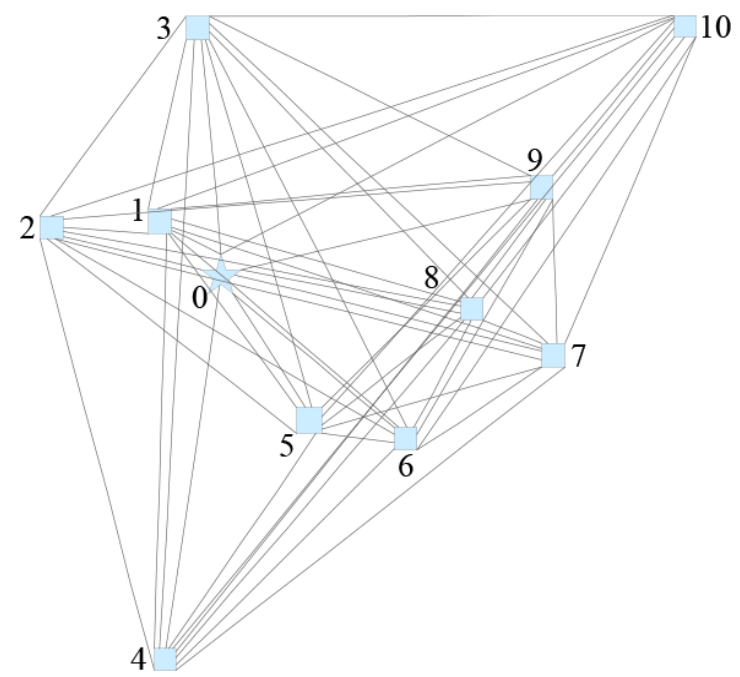

Figure 7. Transportation network topology of the relief dispatching center and all the disaster areas 


\begin{tabular}{|l|c|c|c|c|c|c|c|c|c|c|c|}
\cline { 2 - 12 } \multicolumn{1}{c|}{} & $\mathbf{M}$ & $\boldsymbol{P}_{1}$ & $\boldsymbol{P}_{2}$ & $\boldsymbol{P}_{3}$ & $\boldsymbol{P}_{4}$ & $\boldsymbol{P}_{5}$ & $\boldsymbol{P}_{6}$ & $\boldsymbol{P}_{7}$ & $\boldsymbol{P}_{8}$ & $\boldsymbol{P}_{9}$ & $\boldsymbol{P}_{10}$ \\
\hline $\mathbf{M}$ & 0 & $25(0.5)$ & $58(0.9)$ & $85(1.4)$ & $127(1.6)$ & $60(1)$ & $85(1.2)$ & $115(1.5)$ & $95(1.3)$ & $112(1.5)$ & $145(1.8)$ \\
\hline $\boldsymbol{P}_{1}$ & $25(0.5)$ & 0 & $40(0.7)$ & $55(0.9)$ & $134(1.8)$ & $85(1.2)$ & $108(1.4)$ & $120(1.6)$ & $105(1.4)$ & $110(1.4)$ & $162(2)$ \\
\hline $\boldsymbol{P}_{\mathbf{2}}$ & $58(0.9)$ & $40(0.7)$ & 0 & $78(1.2)$ & $130(1.7)$ & $92(1.3)$ & $125(1.6)$ & $134(1.7)$ & $107(1.4)$ & $134(1.8)$ & $198(2.5)$ \\
\hline $\boldsymbol{P}_{3}$ & $85(1.4)$ & $55(0.9)$ & $78(1.2)$ & 0 & $206(2.6)$ & $108(1.4)$ & $134(1.8)$ & $130(1.8)$ & $112(1.6)$ & $95(1.4)$ & $133(1.7)$ \\
\hline $\boldsymbol{P}_{4}$ & $127(1.6)$ & $134(1.8)$ & $130(1.7)$ & $206(2.6)$ & 0 & $90(1.3)$ & $113(1.5)$ & $142(1.8)$ & $130(1.7)$ & $179(2.3)$ & $249(3.2)$ \\
\hline $\boldsymbol{P}_{5}$ & $60(1)$ & $85(1.2)$ & $92(1.3)$ & $108(1.4)$ & $90(1.3)$ & 0 & $35(0.6)$ & $67(1)$ & $55(1)$ & $110(1.3)$ & $153(1.9)$ \\
\hline $\boldsymbol{P}_{6}$ & $85(1.2)$ & $108(1.4)$ & $125(1.6)$ & $134(1.8)$ & $113(1.5)$ & $35(0.6)$ & 0 & $60(1)$ & $51(0.9)$ & $90(1.1)$ & $138(1.7)$ \\
\hline $\boldsymbol{P}_{7}$ & $115(1.5)$ & $120(1.6)$ & $134(1.7)$ & $130(1.8)$ & $142(1.8)$ & $67(1)$ & $60(1)$ & 0 & $25(0.5)$ & $58(0.9)$ & $106(1.3)$ \\
\hline $\boldsymbol{P}_{\mathbf{8}}$ & $95(1.3)$ & $105(1.4)$ & $107(1.4)$ & $112(1.6)$ & $130(1.7)$ & $55(1)$ & $51(0.9)$ & $25(0.5)$ & 0 & $45(1.0)$ & $92(1.5)$ \\
\hline $\boldsymbol{P}_{9}$ & $112(1.5)$ & $110(1.4)$ & $134(1.8)$ & $95(1.4)$ & $179(2.3)$ & $110(1.3)$ & $90(1.1)$ & $58(0.9)$ & $45(1.0)$ & 0 & $75(1.1)$ \\
\hline $\boldsymbol{P}_{10}$ & $135(1.6)$ & $162(2)$ & $198(2.5)$ & $133(1.7)$ & $249(3.2)$ & $153(1.9)$ & $138(1.7)$ & $106(1.3)$ & $92(1.5)$ & $75(1.1)$ & 0 \\
\hline
\end{tabular}

Table 3. Running distance $D_{i j}$ and running time $T_{i j}$ of vehicles

\begin{tabular}{|c|c|c|c|c|c|c|c|c|c|c|}
\cline { 2 - 8 } & $\boldsymbol{P}_{1}$ & $\boldsymbol{P}_{\mathbf{2}}$ & $\boldsymbol{P}_{3}$ & $\boldsymbol{P}_{4}$ & $\boldsymbol{P}_{5}$ & $\boldsymbol{P}_{6}$ & $\boldsymbol{P}_{7}$ & $\boldsymbol{P}_{8}$ & $\boldsymbol{P}_{9}$ & $\boldsymbol{P}_{10}$ \\
\hline $\boldsymbol{M R}_{\boldsymbol{j}}$ & 8 & 6 & 1 & 2 & 10 & 12 & 17 & 21 & 15 & 6
\end{tabular}

Table 4. Quantity of cables that disaster areas require

Based on the evaluation method about the urgency of the requirement for reliefs in different disaster areas proposed in this paper, we analyze on the urgency of every disaster area. For the disaster area $P_{1}$, according to the method proposed by Guo et al. (2013), the subjective weights of the indexes are calculated through the method of AHP, and the subjective weight set $\boldsymbol{\alpha}=(0.168,0.156,0.076,0.336,0.084,0.180)$ is got; according to the method proposed by Guo et al. (2014), the objective weights of the indexes are calculated through the Entropy Method, and the objective weight set $\boldsymbol{\beta}=(0.124,0.209,0.022,0.412,0.101,0.132)$ is got; based on the subjective and objective weight set, we calculate and get the comprehensive weight set $\boldsymbol{U}^{\prime}=(0.147,0.183,0.042,0.378,0.094,0.157)$ by the Equation (1) and Equation (2). Then, 5 experts are invited to evaluate on each index and all the experts' evaluation results are combined by the improved evidence combination method proposed in this paper. Based on this, we get the fuzzy evaluation matrix which is shown in the Equation (38). Finally, we combine the weights of all the indexes with the fuzzy evaluation matrix by the weighted composition operator and get $\chi_{1}^{\prime}=0.390$ which is the evaluation value of the urgency of the requirement for reliefs in the disaster area $P_{1}$. Similarly, we calculate and get the evaluation values of the urgency of the requirement for reliefs in other disaster areas, which are shown in Table 5. 


$$
R=\left|\begin{array}{lll}
0.007 & 0.234 & 0.759 \\
0.014 & 0.562 & 0.424 \\
0.245 & 0.326 & 0.429 \\
0.109 & 0.409 & 0.482 \\
0.431 & 0.454 & 0.115 \\
0.272 & 0.525 & 0.293
\end{array}\right|
$$

\begin{tabular}{|c|c|c|c|c|c|c|c|c|c|c|}
\cline { 2 - 9 } & $\boldsymbol{P}_{\mathbf{1}}$ & $\boldsymbol{P}_{\mathbf{2}}$ & $\boldsymbol{P}_{3}$ & $\boldsymbol{P}_{4}$ & $\boldsymbol{P}_{5}$ & $\boldsymbol{P}_{6}$ & $\boldsymbol{P}_{7}$ & $\boldsymbol{P}_{8}$ & $\boldsymbol{P}_{9}$ & $\boldsymbol{P}_{\mathbf{1 0}}$ \\
\hline$\chi_{j}^{\prime}$ & 0.390 & 0.225 & 0.105 & 0.198 & 0.474 & 0.536 & 0.723 & 0.856 & 0.673 & 0.279 \\
\hline
\end{tabular}

Table 5. Evaluation value of the urgency of the requirement for reliefs in different disaster areas

Based on the adaptive Genetic Algorithm proposed in this paper, we solve the emergency dispatch model considering the urgency of the requirement for reliefs in different disaster areas. Set popsize $=50, D=500, P_{c \max }=0.9, P_{c \min }=0.4, P_{m \max }=0.1, P_{m \min }=0.01$. The weights of objective function $f_{1}, f_{2}, f_{3}$ are calculated based on the method proposed by Zhang et al. (2014), and the results are $\lambda_{1}=0.553, \lambda_{2}=0.109, \lambda_{3}=0.338$. The algorithm is implemented by Matlab R2010b and ran on PCs with $2.4 \mathrm{GHz}$ and 4GB RAM memory. After implementation, we can get the changing curve of the fitness values as shown in Figure 8 and the emergency dispatch scheme as shown in Figure 9. In Figure 9, the numerical values in the brackets represent the quantity of the reliefs distributed to the disaster areas.

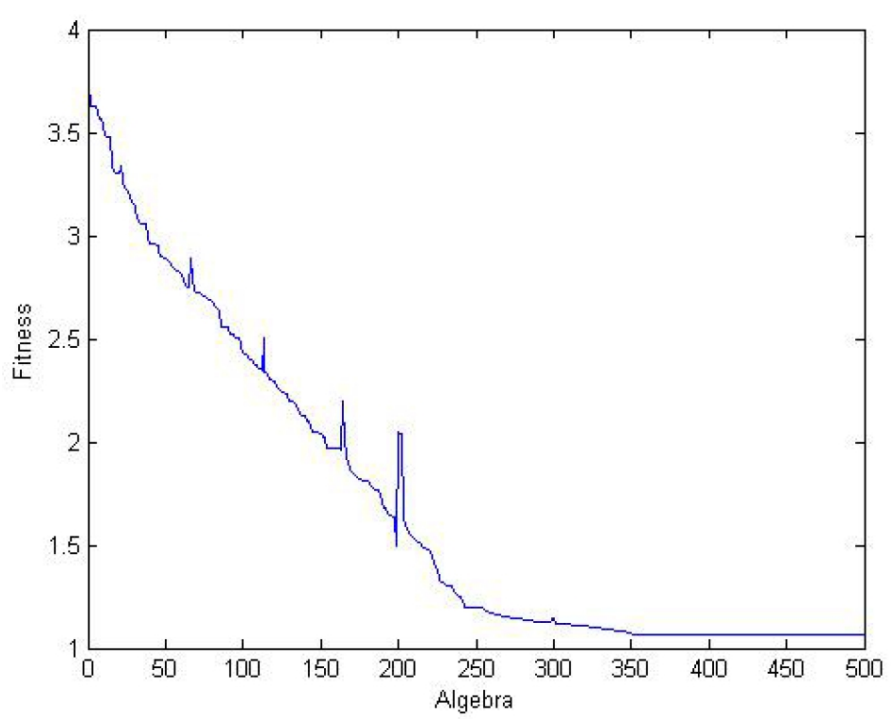

Figure 8 . Changing curve of the fitness values 


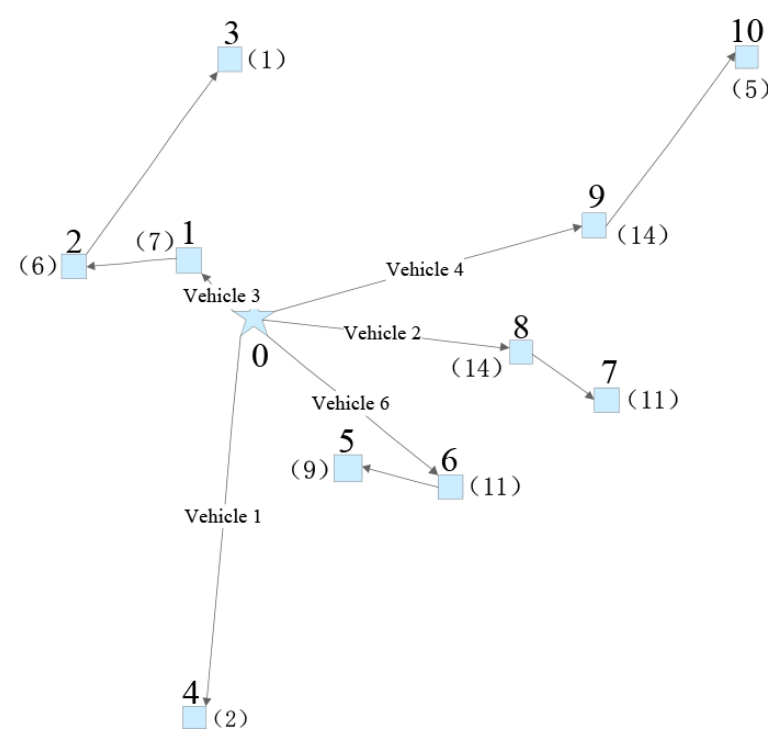

Figure 9. Emergency dispatch scheme considering the urgency of the requirement for reliefs in different disaster areas

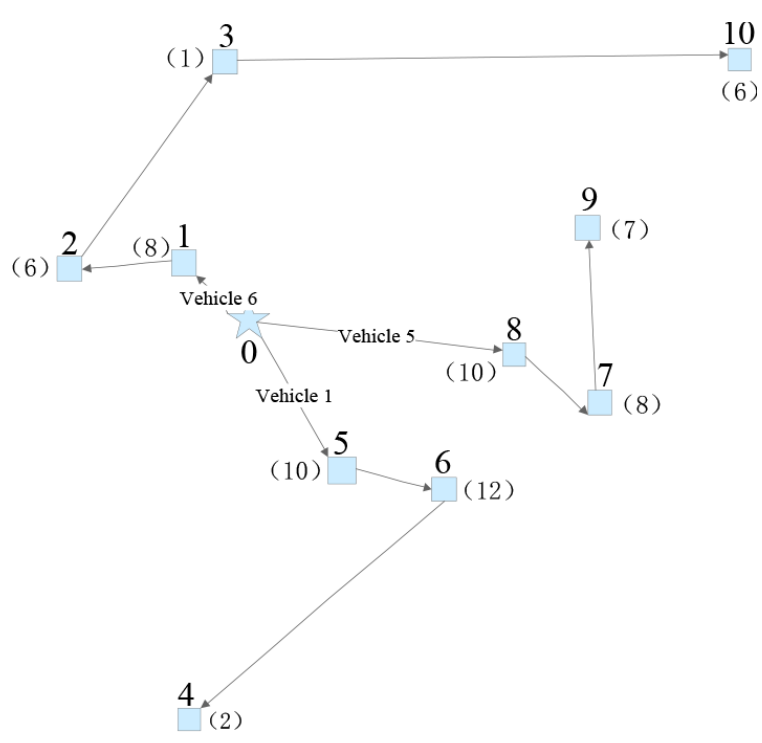

Figure 10. Emergency dispatch without the urgency of the requirement for reliefs in different disaster areas

From Figure 8 we can know that as the evolutional generation increases, the fitness value gradually becomes stable and converges to the most optimal solution in generation 355 , at this time, the fitness value is 1.065 . From Figure 9 we can know that the running routes of vehicles meet the distribution priority requirement of disaster area with the higher urgency.

Furthermore, in order to analyze the influence of the urgency of the requirement for reliefs in different disaster areas on the emergency dispatch scheme, based on the model proposed by Chang et al.(2014), we calculate and get the emergency dispatch scheme without the consideration of the urgency of the requirement for reliefs in different disaster areas. This emergency dispatch scheme is shown in Figure 10 and is compared with the scheme calculated based on the model proposed in this paper. The comparative analysis results are shown in Table 6.

\begin{tabular}{|l|r|r|r|}
\cline { 2 - 4 } & \multicolumn{1}{|c|}{ Response time } & Distribution cost & $\begin{array}{c}\text { Unsatisfied rate of } \\
\text { requirement for reliefs }\end{array}$ \\
\hline This paper & 15.9 & 19020 & 1.228 \\
\hline Chang et al. (2014) & 19.4 & 15160 & 2.26 \\
\hline
\end{tabular}

Table 6. Comparative analysis on the emergency dispatch scheme based on the different models

From Figure 9 and Figure 10 we can know that under the condition that the urgency of the requirement for reliefs in different disaster areas is not considered, the running routes of some 
vehicles (for example, the running routes $0 \rightarrow 5 \rightarrow 6 \rightarrow 4$ ) violate the realistic requirement that the disaster area with the higher urgency has a priority to obtain the reliefs. However, the model proposed in this paper can better meet the realistic requirement to guarantee the timeliness of relief supply, and then to take advantage of the rescue value of reliefs.

From Table 6 we can know that the emergency dispatch scheme got based on the model proposed in this paper has a shorter response time and higher satisfied rate of requirement for reliefs. This emergency dispatch scheme has a higher distribution cost compared with the scheme got based on the model proposed by Chang et al.(2014). However, due to the weak economy of emergency dispatch, compared with the timeliness of relief supply, distribution cost becomes less important. After comprehensive analysis, it is the emergency dispatch model considering the urgency of the requirement for reliefs in different disaster areas proposed in this paper more reasonable.

\section{Conclusion}

In order to dispatch reliefs in emergency circumstances reasonably, we propose an emergency dispatch model aiming to minimize the response time, the distribution cost and the unsatisfied rate of the requirement for reliefs based on the urgency of the requirement for reliefs in different disaster areas. Considering that the information that decision-makers obtain when sudden-onset disasters occur is fuzzy and uncertain, we put forward to an evaluation method based on fuzzy comprehensive evaluation and improved evidence theory to quantify the urgency of the requirement for reliefs in different disaster areas. Then, we improve the Genetic Algorithm based on adaptive crossover and mutation probability function to solve the emergency dispatch model. Finally, the case that the $Y$ hydraulic power enterprise carries on emergency dispatch of reliefs under continuous sudden-onset heavy rain is used to verify the availability of the proposed emergency dispatch model. The results show that the emergency dispatch model meets the distribution priority requirement of disaster area with the higher emergency degree, so that reliefs are supplied more timely.

The problem of emergency dispatch under sudden-onset disasters is a complex optimization problem. The quantity of reliefs that disaster area requires and the running time of vehicles are viewed as available information, and the problem that how to obtain the information is not considered. In fact, due to the unexpectedness of disasters, the quantity of reliefs required usually is fuzzy. Besides, due to the destructiveness of disasters, the conditions of transportation roads are uncertain, and then the running time of vehicles are usually uncertain. Therefore, dealing with the fuzzy and uncertain information in the process of emergency dispatch effectively will be the further research. 


\section{Acknowledgment}

Project supported by the National Natural Science Foundation, China (No.71571023); Specialized Research Fund for the Doctoral Program of Higher Education (No.20130191120001); Project supported by China Scholarship Council (No.[2014]3072).

\section{References}

Bao, B-F., Yang, Y., Li, L-T., Li, F., Liu, A-J., \& Liu, N. (2014). Importance evaluation method for innovative customer in collaborative products innovation. Computer Integrated Manufacturing Systems, 04, 739-746.

Barbarosoglu, G., \& Arda Y. (2002). A two-stage stochastic programming framework for transportation planning in disaster response. Journal of the Operational Research, 140(1), 118-133.

Bozorgi-Amiri, A., Jabalameli, M.S., \& Alinaghian, M. (2011). A modified particle swarm optimization for disaster relief logistics under uncertain environment. The International Journal of Advanced Manufacturing Technology, 60(1-4), 357-371. http://dx.doi.org/10.1007/s00170-011-3596-8

Chang, F-S., Wu, J-S., \& Lee, C-N. (2014). Greedy-search-based multi-objective genetic algorithm for emergency logistics scheduling. Expert Systems with Applications, 41(6), 2947-2956. http://dx.doi.org/10.1016/j.eswa.2013.10.026

Gao, S-S., Niu, L-Q., \& Yang, K. (2009). A New and Better Rule for Combining Sharply Conflicting Evidences. Journal of North Western Poly Technical University, 27(1), 43-46.

Ge, H-L., Liu, N., Zhang, G-C., \& Yu H-H. (2010). A model for distribution of multiple emergency commodities to multiple affected areas based on loss of victims of calamity. Journal of Systems \& Management, 05, 541-545.

George, T., \& Pal, N.R. (1996). Quantification of conflict in Dempster-Shafer framwork: a new approach. Int. J. Gen. Syst., 24(4), 407-423. http://dx.doi.org/10.1080/03081079608945130

Guo, J-W., Pu, X-Q., Gao, X., \& Zhang, Y-A. (2014). Improved method on weights determination of indexes in multi-objective decision. Journal Of Xidian University, 06, 135142. 
Guo, W., Tong, K-N., Shao, H-Y., Wang, L., \& Zheng, Q. (2013). Small and medium-sized enterprises multi-service agent credit rating system construction under cloud manufacturing mode based on RS and AHP [J]. Computer Integrated Manufacturing Systems, 09, 23402347.

Guo, Z-X., \& Zhang, Q. (2009). Application of fuzzy clustering analysis in classification of emergency materials. Computer Engineering and Applications, 35, 208-211.

International Red Cross. (2014). World disasters report. Geneva: International Red Cross.

Jiang, B., \& Yu, H. (2012). Environmental Impact Assessment of Life-cycle Building Products Using the Evidence Reasoning Approach. International Journal of Networking and Virtual Organisations, 01, 319-333.

Jousselme, A-L., Grenier, D., \& Bosse, E. (2001). A new distance between two bodies of evidence. Information Fusion, 1(2), 91-101. http://dx.doi.org/10.1016/S1566-2535(01)00026-4

Kemball-Cook, D., \& Stephenson, R. (1984). Lessons in logistics from Somalia. Disasters, 8(1), 57-66. http://dx.doi.org/10.1111/j.1467-7717.1984.tb00853.x

Lefevre, E., Colot, O., \& Vannoonrenberghe, P. (2002). Belief functions combination and conflict management. Information Fusion, 3(2), 149-162. http://dx.doi.org/10.1016/S1566-2535(02)00053-2

Li, J., Zhang, J-H., \& Zhu, D-L. (2011). Multi-resource emergency scheduling model and algorithm in disaster chain. Systems Engineering-Theory \& Practice, 31(3), 488-495.

Liu, X-Z., \& Zhou, M. (2009). Improved Adaptive Genetic Algorithm and Its Application to Backward Analysis of Geotechnical Engineering. Journal Of Tongji University (Natural Science), 03, 303-307.

Pang, H-Y., Liu, N., \& Wu, Q. (2012). Decision-making model for transportation and distribution of emergency materials and its modified particle swarm optimization algorithm. Control and Decision, 27(6), 871-874.

Sun, Q., Ye, X-Q., \& Gu, W-K. (2000). A New Combination Rules of Evidence Theory. Acta Electronica Sinica, 28(8), 117-119.

Shu, Q-L. (2012). Resource Allocation and Scheduling for Unconventional Emergency Based on "Scenario-Response". University of Science and Technology of China.

Tian, J., Ma, W-Z., Wang, Y-L., \& Wang, K-L. (2011). Emergency supplies distributing and vehicle routes programming based on particle swarm optimization. Systems EngineeringTheory \& Practice, 31(5), 898-906. 
Vitoriano, B., Ortuno, M., \& Tirado, G. (2011). A multi-criteria optimization model for humanitarian aid distribution. Journal of Global optimization, 51(2), 189-208. http://dx.doi.org/10.1007/s10898-010-9603-z

Wang, H-J., Wang, J., \& Ma, S-H. (2014). Decision-Making for Emergency Materials Dynamic Dispatching Based on Fuzzy Demand and Supply. Chinese Journal of Management Science, 22(1), 55-64.

Wang, X-P., Ma, C., \& Ruan, J-H. (2013). Model and algorithm of relief materials dynamic scheduling without sufficient vehicle quantity. Systems Engineering-Theory \& Practice, $33(6), 1492-1500$.

Wang, X-P., Yang, D-L., \& Xu, C-L. (2009). Study on Disruption Management for Vehicle Routing Problem with the Request Changes of Customer. Operations Research And Management Science, 18(4), 16-24.

Yager, R.R. (1987). On the D-S framework and new combination rules. Information Sciences, 41(2), 93-138. http://dx.doi.org/10.1016/0020-0255(87)90007-7

Yuan, Y., \& Wang, D.W. (2009). Path selection model and algorithm for emergency logistics management. Computers and Industrial Engineering, 56(3), 1081-1094. http://dx.doi.org/10.1016/j.cie.2008.09.033

Zhang, H-J., Zhang, J., Luo, H., \& Xie X-P. (2014). Objective weight computation based on personal preference for multi-objective optimization problem. Control and Decision, 29(8), 1471-1496.

Journal of Industrial Engineering and Management, 2015 (www.jiem.org)

Article's contents are provided on an Attribution-Non Commercial 3.0 Creative commons license. Readers are allowed to copy, distribute and communicate article's contents, provided the author's and Journal of Industrial Engineering and Management's names are included. 